\title{
Structural brain alterations of Down's syndrome in early childhood evaluation by DTI and volumetric analyses
}

\author{
Hediye Pinar Gunbey ${ }^{1}$ - Meltem Ceyhan Bilgici ${ }^{1}$ - Kerim Aslan ${ }^{1}$ • Arzu Ceylan Has ${ }^{2}$. \\ Methiye Gonul Ogur ${ }^{3} \cdot$ Aslihan Alhan $^{4} \cdot$ Lutfi Incesu $^{1}$
}

Received: 12 June 2016 /Revised: 26 August 2016 / Accepted: 29 September 2016 / Published online: 31 October 2016

(C) European Society of Radiology 2016

\begin{abstract}
Objectives To provide an initial assessment of white matter (WM) integrity with diffusion tensor imaging (DTI) and the accompanying volumetric changes in WM and grey matter (GM) through volumetric analyses of young children with Down's syndrome (DS).

Methods Ten children with DS and eight healthy control subjects were included in the study. Tract-based spatial statistics (TBSS) were used in the DTI study for whole-brain voxelwise analysis of fractional anisotropy (FA) and mean diffusivity (MD) of WM. Volumetric analyses were performed with an automated segmentation method to obtain regional measurements of cortical volumes.

Results Children with DS showed significantly reduced FA in association tracts of the fronto-temporo-occipital regions as well as the corpus callosum (CC) and anterior limb of the internal capsule $(\mathrm{p}<0.05)$. Volumetric reductions included total cortical $\mathrm{GM}$, cerebellar GM and WM volume, basal ganglia, thalamus, brainstem and CC in DS compared with controls $(\mathrm{p}<0.05)$. Conclusion These preliminary results suggest that DTI and volumetric analyses may reflect the earliest complementary changes of the neurodevelopmental delay in children with
\end{abstract}

Hediye Pınar Gunbey

hpgunbey@hotmail.com

1 Faculty of Medicine, Department of Radiology, Ondokuz Mayıs University, Neuroradiology Section, 55139 Kurupelit, Samsun, Turkey

2 National Magnetic Resonance Research Center, Bilkent University, Ankara, Turkey

3 Department of Genetics, Ondokuz Mayıs University, Samsun, Turkey

4 Department of Statistics, Ufuk University, Ankara, Turkey
DS and can serve as surrogate biomarkers of the specific elements of WM and GM integrity for cognitive development. Key Points

- DS is the most common genetic cause of intellectual disability.

-WM and GM structural alterations represent the neurological features of DS.

- DTI may identify the earliest aging process changes.

- DTI-volumetric analyses can serve as surrogate biomarkers of neurodevelopment in DS.

Keywords Down's syndrome · Brain · MRI · DTI · Volumetry

\section{Introduction}

Down's syndrome (DS), or trisomy 21, is the most common genetic cause of intellectual disability and occurs in 14.7 out of every 10,000 live births [1]. The association of several neurological features, such as impairments in language [2], cognition [3], learning and memory [4], with unclear origin make it one of the most speculated neurodevelopmental disorders in childhood. The cognitive profiles of DS include language deficits in articulation [5], syntactic weakness [6] and significant verbal short-term memory deficits [7] accompanied by motor weaknesses [8].

Although the clinical features of DS have been characterized, few neuroimaging studies have qualified the pathophysiology of the neurological deficits in DS. As the first manifestation of neuroanatomical abnormality, a characteristic reduced brain size of children with DS appears in the 4- to 5-month fetus and progresses during the last 3 months of gestation $[9$, 10]. Structural MRI reports, based on fewer than 15 original studies in children and youths, have suggested that the total 
brain volume is smaller [11-14], with specific reductions in cerebellar [11-13, 15] and hippocampal volumes [12, 14, 15]. Additionally, several studies have reported regional reductions in the frontal and temporal lobes $[13,14]$ and preserved parietal lobar grey matter (GM) volume [12] in children. Knowledge of GM involvement has been extended by recent paediatric voxelbased morphometry (VBM) studies with high spatial resolutions. Differences in GM density have been reported in the frontal temporal lobes and cerebellum [15-17]. The latest study utilizing cortical morphometry in young people also noted reductions of cortical GM and white matter (WM) volumes, accompanied by decreased frontotemporal surface areas [18]. A case study of cortical morphometry using FreeSurfer reported reduced volumes of bilateral precentral and cingulate gyri [19]. FreeSurfer is a powerful tool with high reproducibility and accuracy for extensive and automated analysis of the human brain [20]; it provides completely automated parcellation of the cerebral cortex and subcortical structures.

Due to the severity of the DS cognitive phenotypes, it is surprising that no existing study has evaluated the WM integrity of children with DS. The impaired intellectual function in DS is caused by abnormalities in memory, language and learning [21]. Morphosyntax, verbal short-term memory and explicit long-term memory are typically affected by memory and language abilities. Along with volume reductions, the connections of white matter structures between the frontal and temporal lobes may also be affected and may disclose the underlying pathophysiology of these deficits. In addition, emotion and the affected visuospatial functions may also be linked to limbic and temporo-occipital connections. Lastly, an evaluation of cerebellar microstructures may define the involvement of motor and non-motor functions in the cerebellums of patients with DS [22]. Due to the growing interest in potential alterations in anatomical connectivity, diffusion tensor imaging (DTI) has become a popular tool for assessing structural connectivity in the human brain.

DTI is a non-invasive, advanced MRI method that characterises the microstructure of WM in vivo by quantitatively measuring the diffusion of water [23]. DTI parameters, especially fractional anisotropy (FA), identify the directional diffusion properties of tissues and are sensitive to anomalies in axonal density, diameter and myelination within WM tracts [24]. Another DTI parameter, mean diffusivity (MD), reflects the magnitude of diffusion that is independent from anisotropy and demonstrates the degrees of myelination, interstitial space and axonal density. According to the existing literature, only one DTI study has evaluated the WM integrity of DS in adults: Powell et al. [25] reported FA decline in evaluating dementia in DS adults. Against the background of morphological brain changes that have been detected as early as in 4- to 5-month fetuses, we aimed to investigate 2- to 4-year-old children with DS. These data provide an initial assessment of WM integrity and accompanying white and grey matter volumetric changes in early DS childhood.

\section{Materials and methods}

\section{Subjects}

The study included ten children with DS (five girls and five boys; mean age $2.6 \pm 0.69$ years) and eight healthy controls (four girls and four boys; mean age $2.5 \pm 0.707$ years). The patients were recruited from DS individuals who were referred for brain MRI acquisition by the Department of Genetics during clinical follow-up. The diagnosis of DS was confirmed by karyotype examinations that showed trisomy of the chromosome 21. Exclusion criteria included untreated medical conditions that might affect cognition (e.g. hypothyroidism in DS). The control group consisted of eight right-handed, age- and sex-matched healthy subjects who had been referred for MRI scan for other reasons and had normal findings on structural MRI. Controls were excluded for having any history of previous neurological, psychiatric or systemic disease. After a complete description of the study, informed consent was obtained from the patients' parents before the administration of sedative drugs prior to going for the MR scan. The study was approved by the Ethics Committee of Ondokuz Mayis University Medical School, Samsun, Turkey.

The MRI studies were conducted under sedation with chloral hydrate $(\mathrm{CH})$. The protocol for paediatric MRI sedation was $50 \mathrm{mg} / \mathrm{kg} \mathrm{CH}$, followed by a second dose if sedation failed. In patients who were monitored, oxygen saturation and electrocardiogram monitoring were applied during sedation. The patients were discharged 1-2 hours after they recovered.

\section{Image acquisition and processing}

All images were acquired on a 1.5-T Philips Gyroscan Intera system equipped with a Synergy-L Sensitivity Encoding (SENSE) head coil. The DTI data were acquired using a 16direction EPI sequence with 60 contiguous 2-mm AC-PC aligned interleaved slices with no gap $(\mathrm{TR} / \mathrm{TE}=10.15 /$ $90 \mathrm{~ms} ;$ matrix $=128 \times 128 ; \mathrm{b}$-value $=1,000 ; \mathrm{FOV}=256 \mathrm{~mm}$, 2 -mm isotropic resolution, NSA $=3$ ). In addition, a high resolution T1-weighted $3 \mathrm{D}$ gradient-echo sequence $(\mathrm{TR} / \mathrm{TE}=$ $7.2 / 33 \mathrm{~ms} ;$ matrix $=256 \times 256$ pixels $; \mathrm{NSA}=1 ; \mathrm{FOV}=$ $256 \mathrm{~mm}$; slice thickness $=1 \mathrm{~mm}$; gap $=0 \mathrm{~mm}$; flip angle $=$ $8^{\circ}$ ) of structural images of 160 slices of the entire brain was acquired for anatomical reference.

DTI data were analysed using FSL v. 4.1 .5 (Functional MRI of the Brain software library, FMRIB). Preprocessing of the raw images of diffusion-weighted data involved correcting of head motion, eddy current distortion and diffusion tensor fitting using FMRIB's Diffusion Toolbox (FDT v. 2.0). After pre-processing, FA and MD were calculated. Tractbased spatial statistics (TBSS) (TBSS v. 1.2; http://www. fmrib.ox.ac.uk/fsl/ tbss/) were used for voxelwise statistical analyses of the DTI data. The thinned mean FA skeleton 
was computed after registration of FA maps and aligning to the average space as input for TBSS. A permutation-based, non-parametric test was performed with 500 permutations to produce the voxelwise statistics.

The threshold-free cluster enhancement was used to avoid the use of an arbitrary threshold in the initial cluster formation and output was corrected for multiple comparisons. Familywise error corrected maps were obtained with p-values less than 0.05. Standard cluster-based thresholding corrected for multiple comparisons, comprising Gaussian-smoothing, thresholding of the smoothed data at a cluster-forming $\mathrm{t}$ threshold of two and, finally, formation of the contiguous clusters of supra-threshold voxels (using 26-neighbour connectivity) were carried out. Supratentorial WM clusters with significant changes on the resulting TBSS maps were extracted as regions of interest (ROIs) and registered and overlaid onto an anatomical Montreal Neurological Institute (MNI) template. These ROIs were labelled according to Johns Hopkins University WM tractography and the International Consortium for Brain Mapping DTI-81 WM atlases in FSL, and the mean diffusion indices of the ROIs were calculated. The author who performed the measurements was blind to subjects' clinical information and compared right and left sides. For the special analyses for measuring FA values, ROIs were drawn to the right cerebral peduncle (R-CP), bilateral posterior limb of internal capsule (PLIC), uncinate fasciculus (UF), inferior longitudinal fasciculus (ILF), inferior fronto-occipital fasciculus (IFOF), cingulum (CIN) and corpus callosum minor forceps (CCminF) (Fig. 1).

The volumetric segmentation analysis was performed using Freesurfer v. 5.3.0 (http://surfer.nmr.mgh.harvard. $\mathrm{edu} /$ ) including the removal of non-brain tissue (skull, eyeballs and skin), using an automated algorithm with the ability to successfully segment the whole brain without any user intervention. Next, cortical surface reconstruction methods were applied to acquire regional measurements of cortical volume $\left(\mathrm{mm}^{3}\right)$. Fischl et al. [26] described the automated procedures for volumetric measurements of the different structures of the brain. After checking the reconstructed cortical surface models to confirm segmentation accuracy for each participant, regions with poor segmentation accuracy were excluded from analyses due to poor image quality or misregistration. Cortical surfaces were automatically parcellated and combined to create averages for cortical-total GM and for frontal, temporal, parietal and occipital lobar regions.

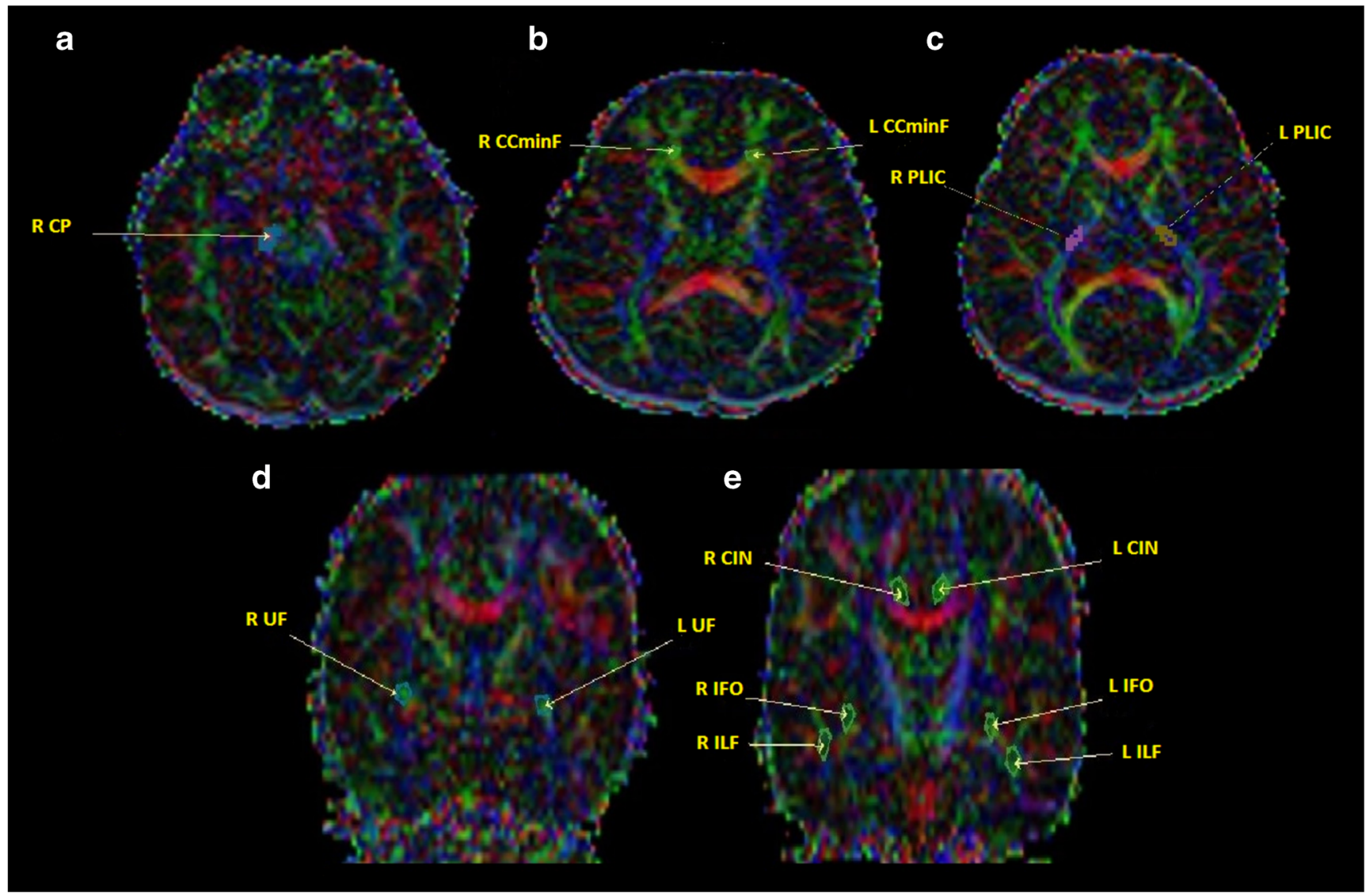

Fig. 1 The regions of interest (ROIs) on coloured fractional anisotropy (FA) maps on axial (A, B, C) and coronal sections (D, E). $R$ right, $L$ left, $C P$ cerebral peduncle, $P L I C$ posterior limb of internal capsule, IFOF inferior frontooccipital fasciculus, $I L F$ inferior longitudinal fasciculus, $U F$ uncinate fasciculus, $C I N$ cingulum, $C C \min F$ corpus callosum minor forceps 
The Shapiro-Wilk test was used to determine normality, and the Mann-Whitney $U$ test was used for comparisons of the means of variables with controls. Correlations between volumetric values and volumetric-DTI values were calculated with Spearman's correlation test. A p-value less than 0.05 was considered to indicate statistical significance.

\section{Results}

\section{Volumetric analyses}

Children with DS showed significant reductions in the regional GM volume of of the left putamen, bilateral thalamus proper, caudate nucleus and cerebellar cortex, as well as the brain stem and corpus callosum $(\mathrm{CC})(\mathrm{p}<0.05)$. Additionally, subcortical GM volume (SGMV) and total cortical GM volume (TCGMV) of both hemispheres and right cerebellar WM volume were significantly lower in DS subjects compared to controls $(\mathrm{p}<0.05)$ (Table 1$)$. There were no significant differences in the total WM, total GM and brain segmentation volumes $(\mathrm{p}>0.05)$ between DS subjects and the controls.

\section{DTI analyses}

The whole brain TBSS revealed extensive reductions of FA in the supratentorial WM including right-CP, right-UF, CC body, bilateral ILF/IFOF and right anterior limb of the internal capsule (ALIC). Clusters showing increments of MD values for DS compared to control subjects were found in the CP, ILF and IFOF of the right hemisphere and in the external capsule and posterior thalamic radiation (PTR) of the left hemisphere.
Table 1 Regional brain volumes of subjects with Down's syndrome versus control subjects

\begin{tabular}{|c|c|c|c|}
\hline Region & $\begin{array}{l}\text { Children with Down's } \\
\text { syndrome }(\mathrm{N}=10) \\
\text { mean } \pm \mathrm{SD}\end{array}$ & $\begin{array}{l}\text { Control group } \\
(\mathrm{N}=8) \\
\text { mean } \pm \mathrm{SD}\end{array}$ & $\mathrm{p}$-value \\
\hline \multicolumn{4}{|l|}{ Volume measures $\left(\mathrm{cm}^{3}\right)$} \\
\hline Brain segmentation volume & $860.2 \pm 50.4$ & $845.4 \pm 44.4$ & 0.624 \\
\hline R CGMV & $219.2 \pm 16.1$ & $234.1 \pm 18.1$ & $0.037 *$ \\
\hline L CGMV & $219.5 \pm 9.2$ & $233.7 \pm 18.3$ & $0.049 *$ \\
\hline T CGMV & $438.6 \pm 25.3$ & $467.8 \pm 37.1$ & $0.037 *$ \\
\hline SC GMV & $41.7 \pm 2.5$ & $45.6 \pm 3.2$ & $0.014 *$ \\
\hline T GMV & $579.3 \pm 42.3$ & $563.2 \pm 26.8$ & 0.270 \\
\hline R WMV & $125.8 \pm 9.3$ & $128.9 \pm 10.8$ & 0.462 \\
\hline L WMV & $124.7 \pm 9.3$ & $128.4 .6 \pm 1.0$ & 0.327 \\
\hline T WMV & $250.5 \pm 18.5$ & $257.3 .6 \pm 20.7$ & 0.327 \\
\hline $\mathrm{R}$ thalamus & $5.0 \pm 0.2$ & $5.4 \pm 0.2$ & $0.014 *$ \\
\hline L thalamus & $4.9 \pm 0.1$ & $5.3 \pm 0.3$ & $0.010^{*}$ \\
\hline $\mathrm{R}$ caudate & $3.0 \pm 0.1$ & $3.3 \pm 0.4$ & $0.05^{*}$ \\
\hline L caudate & $2.7 \pm 0.4$ & $3.2 \pm 0.4$ & $0.05^{*}$ \\
\hline R putamen & $5.0 \pm 0.7$ & $4.3 \pm 0.6$ & 0.66 \\
\hline L putamen & $4.5 \pm 0.4$ & $5.0 \pm 0.6$ & $0.05^{*}$ \\
\hline $\mathrm{R}$ pallidum & $1.3 \pm 0.1$ & $1.4 \pm 0.1$ & 0.540 \\
\hline L pallidum & $1.5 \pm 0.09$ & $1.7 \pm 0.2$ & $0.02 *$ \\
\hline $\mathrm{R}$ hippocampus & $2.9 \pm 0.2$ & $2.8 \pm 0.4$ & 0.903 \\
\hline L hippocampus & $2.9 \pm 0.2$ & $3.0 \pm 0.2$ & 0.391 \\
\hline $\mathrm{R}$ amygdala & $1.0 \pm 0.1$ & $1.0 \pm 0.1$ & 0.270 \\
\hline $\mathrm{L}$ amygdala & $1.0 \pm 0.1$ & $1.0 \pm 0.1$ & 0.806 \\
\hline Brain stem & $10.6 \pm 1.1$ & $12.0 \pm 1.3$ & $0.027 *$ \\
\hline $\mathrm{R}$ cer cortex & $5.4 \pm 0.2$ & $42.7 \pm 1.3$ & $0.002 * *$ \\
\hline R cer WMV & $5.6 \pm 0.9$ & $7.9 \pm 0.9$ & $0.007 * *$ \\
\hline L cer cortex & $33.2 \pm 4.0$ & $41.3 \pm 0.8$ & $0.002 * *$ \\
\hline L cer WMV & $7.7 \pm 2.8$ & $7.7 \pm 1.0$ & 0.713 \\
\hline
\end{tabular}

$R$ right, $L$ left, $T$ total, $G M V$ grey matter volume, $C G M V$ cortical grey matter volume, $S C$ subcortical, $W M V$ white matter volume

*Analysis of variance $\mathrm{p}<0.05 ; * \mathrm{p}<0.01$ 


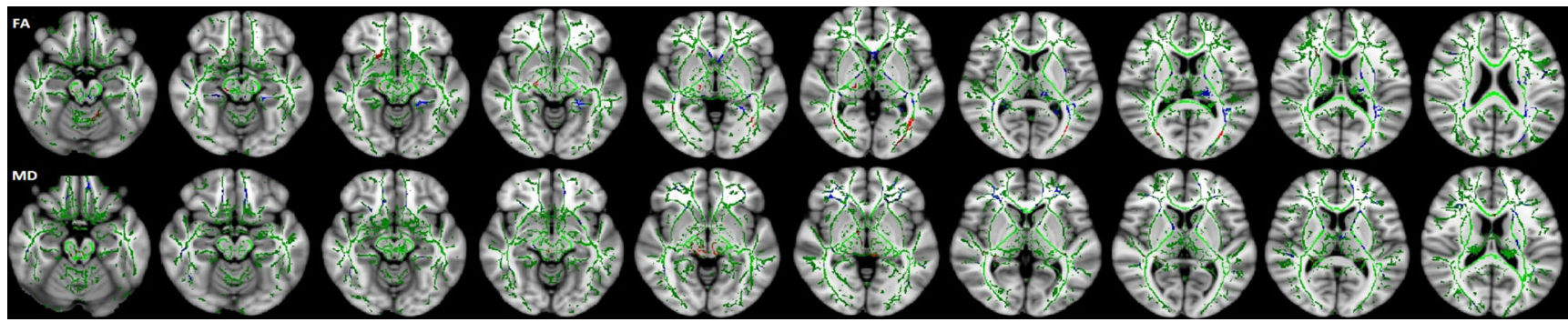

Fig. 2 Tract-based spatial statistics (TBSS) (family-wise error- corrected threshold-cluster extend voxel p maps) display clusters with significantly different fractional anisotropy (FA) and mean diffusivity (MD) of children with Down's syndrome (DS) compared to control participants at $P<0$.05. For all diffusion measures, blue shows increased values and red shows decreased values. FA skeleton projected on a mean FA map is shown in green

right-CIN and right-CP $(\mathrm{p}=0.029, \mathrm{r}=685)$, the right-UF and right-CP $(p=0.024, r=685)$ and the right-ILF and left-IFOF $(\mathrm{p}=0.019, \mathrm{r}=721)$. The left-UC correlated positively with the CCminF in both DS $(\mathrm{p}=0.004, \mathrm{r}=818)$ and control subjects $(\mathrm{p}=0.037, \mathrm{r}=673)$.

\section{Combined volumetry and DTI results}

The correlation analyses of volumetry and DTI values showed a positive $(p=0.016, r=733)$ relationship between FA of the $\mathrm{CCminF}$ and the anterior volume of the $\mathrm{CC}$, which was an expected result. The FA values of the right PLIC correlated positively with the right WM $(\mathrm{p}=0.006, \mathrm{r}=794)$ and total WM volumes $(p=0.005, r=806)$.

\section{Discussion}

The alterations in neuronal and cognitive development with premature aging requires clear understanding of the earliest changes in brain structures for the earliest treatment strategies for DS. Although DS is a neurodevelopmental disorder, most neuroimaging studies have investigated the volumetric analyses of adult and adolescent samples not fit into a narrow age range. Interestingly, no study has investigated the WM integrity of children with DS. The present study revealed preliminary data on alterations in GM and WM integrity through volumetric and DTI analyses in a homogeneous sample of participants between 2 and 4 years old, which is a critical age range for brain overgrowth.

\section{Volumetry}

Results of the present study documented lower widespread GM volumes of SGMV and TCGMV consistent with two recent studies that focused on cortical morphometry in young DS subjects $[18,27]$. A reduced cortical GM volume may reflect the post-mortem findings of simplified gyral patterns, decreased neuronal density, lamination [28] and reduction of about $30-40 \%$ in the number of both neurons and glial cells
Fig. 3 Fractional anisotropy (FA) values of the most affected white matter (WM) tracts from region of interest (ROI) analyses between children with Down's syndrome (DS) and control subjects $(\mathrm{p}<0.05) . R$ right, $L$ left, $C P$ cerebral peduncle, $I L F$ inferior longitudinal fasciculus, $U F$ uncinate fasciculus 
Table 2 Montreal Neurological Institute (MNI) coordinates of selected regions of interest (ROIs) and significant fractional anisotropy (FA) values of both Down's syndrome subjects and controls

\begin{tabular}{lllll}
\hline ROI & MNI coordinates $(\mathrm{x}, \mathrm{y}, \mathrm{z})$ & $\begin{array}{l}\text { Down's syndrome } \\
\text { FA }\end{array}$ & $\begin{array}{l}\text { Control group } \\
\text { FA }\end{array}$ & p-value \\
\hline R CP & $21,-43,-36$ & 0,2482049 & 0,314408125 & 0.033 \\
R PLIC & $24,-18,13$ & 0,4677318 & 0,38414875 & 0,091 \\
L PLIC & $-23,-18,13$ & 0,4924511 & 0,387061375 & 0,062 \\
R IFOF & $35,-10,-12$ & 0,3989686 & 0,479078625 & 0,131 \\
L IFOF & $-36,-14,-12$ & 0,4367896 & 0,48864925 & 0,424 \\
R ILF & $42,-29,-12$ & 0,3679816 & 0,250141 & 0.044 \\
L ILF & $-41,-29,-12$ & 0,2487341 & 0,24346275 & 0,051 \\
R UF & $36,2,-15$ & 0,2727017 & 0,3578645 & 0.016 \\
L UF & $-35,-5,-15$ & 0,390128 & 0,289574875 & 0.006 \\
R CIN & $8,19,29$ & 0,3126787 & 0,2359035 & 0,214 \\
L CIN & $-7,13,29$ & 0,2818979 & 0,200515375 & 0,183 \\
R CC minF & $-7,30,5$ & 0,5488533 & 0,40169375 & 0,155 \\
L CC minF & $9,29,5$ & 0,5390404 & 0,380602625 & 0,131 \\
\hline
\end{tabular}

$R$ right, $L$ left, $R O I$ region of interest, $M N I$ Montreal Neurological Institute, $C P$ cerebral peduncle, $P L I C$ posterior limb of internal capsule, IFOF inferior fronto-occipital fasciculus, ILF inferior longitudinal fasciculus, UF uncinate fasciculus, $C I N$ cingulum, $C C \min F$ corpus callosum minor forceps, $F A$ fractional anisotrophy of neocortical regions in DS [29]. This abnormal development of cortical neurons can lead to learning and memory problems or seizures that begin to emerge in late infancy.

Although a regional GM volume decline in the left putamen, bilateral thalamus and caudate nucleus as well as the brain stem represents initial data in volumetric neuroimaging studies of DS, Anderson et al. [30] reported involvement of these structures with functional MR imaging (fmri) and Karlsen et al. [29] showed there were about $40 \%$ fewer neurons in the thalami of DS brains compared to control brains. Gating sensory input from the brainstem and sensory areas to the cortex and other subcortical regions leads the thalamus and basal ganglia to act as key components for integrating cognition and affective experience. Therefore, these structural alterations may be considered in causative issues of cognitive impairment in DS.

The significantly smaller $\mathrm{CC}$ was consistent with previous findings [31], and reflects the loss of neocortical neuronal projections involved in the maintenance of higher cognitive processes. Our findings of smaller cerebellar volumes were also consistent with the results of prior neuroimaging studies in children with DS $[12,13,32]$. In terms of cortical and WM volume reductions, the results of the current study are concordant with two recent paediatric neuroimaging studies utilizing voxel-based morphometry $[15,17]$. The cerebellum plays a major role in the regulation of proprioceptive motor control and motor learning as verified by the research of Rigoldi et al. [16], reporting a strong relationship between cerebellar vermis volume reduction and quality of gait in patients with DS. The involvement of the cerebellum might contribute to the cognitive phenotypes of DS through the non-motor cortico-cerebellar and cerebellar-limbic circuits that are involved in emotion, attention, working memory, executive control and language learning [33]. Recently, Menghini et al. [17] found significant regional reductions in GM and positive correlations between GM density of cerebellum and measurements of linguistic ability.

Our results did not show as significant a difference in terms of total brain, segmentation and hippocampal volumes as previous reports $[12,14,15]$. The total and subcortical GM volume reduction may not have a significant influence on total brain and hippocampal volume in this age group. A comparison with these studies is challenging due to the wide age range in their included participants.

While we examined the youngest population of DS in neuroimaging studies, they also included young adults, with difficulties in establishing findings on early developmental or neurodegenerative progress.

\section{DTI}

These DTI data represent the first published study of children with DS. We observed significantly lower FA values in a subset of supratentorial WM tracts, which included the right-CP, right-UF, CC body, right ALIC and bilateral ILF and IFOF in DS. Our TBSS study's results were consistent with those of Powell et al.'s DTI study on the frontal WM integrity of adults with DS [25]. As they reported we also detected much of the FA decrease in frontal circuits.

The internal capsule comprises much of the centrum semiovale and courses from the cortex to the brainstem continuing as cerebral peduncles. Its first subdivision ALIC connects the thalamus and prefrontal cortex and contains axons in the frontopontine pathway. ALIC involvement in several 
Volumetric reduction

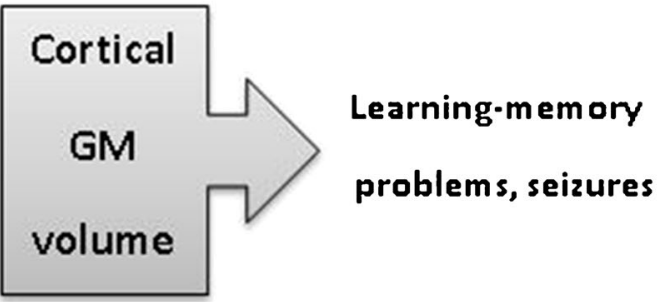

DT-FA reduction
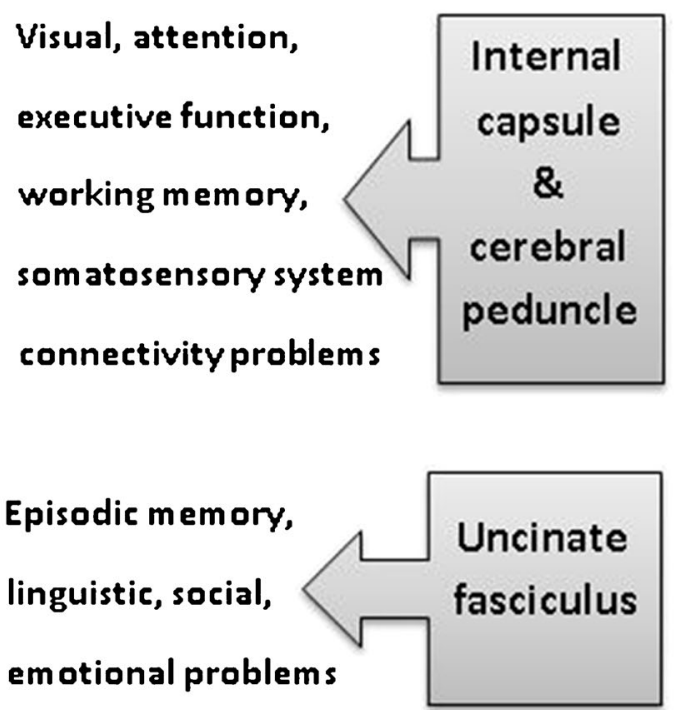

Loss of neuronal

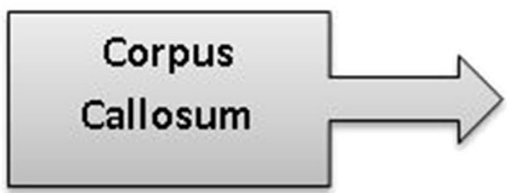

connections $\rightarrow$

Cognitive dysfunction \&

Corpus

Disconnection syndromes
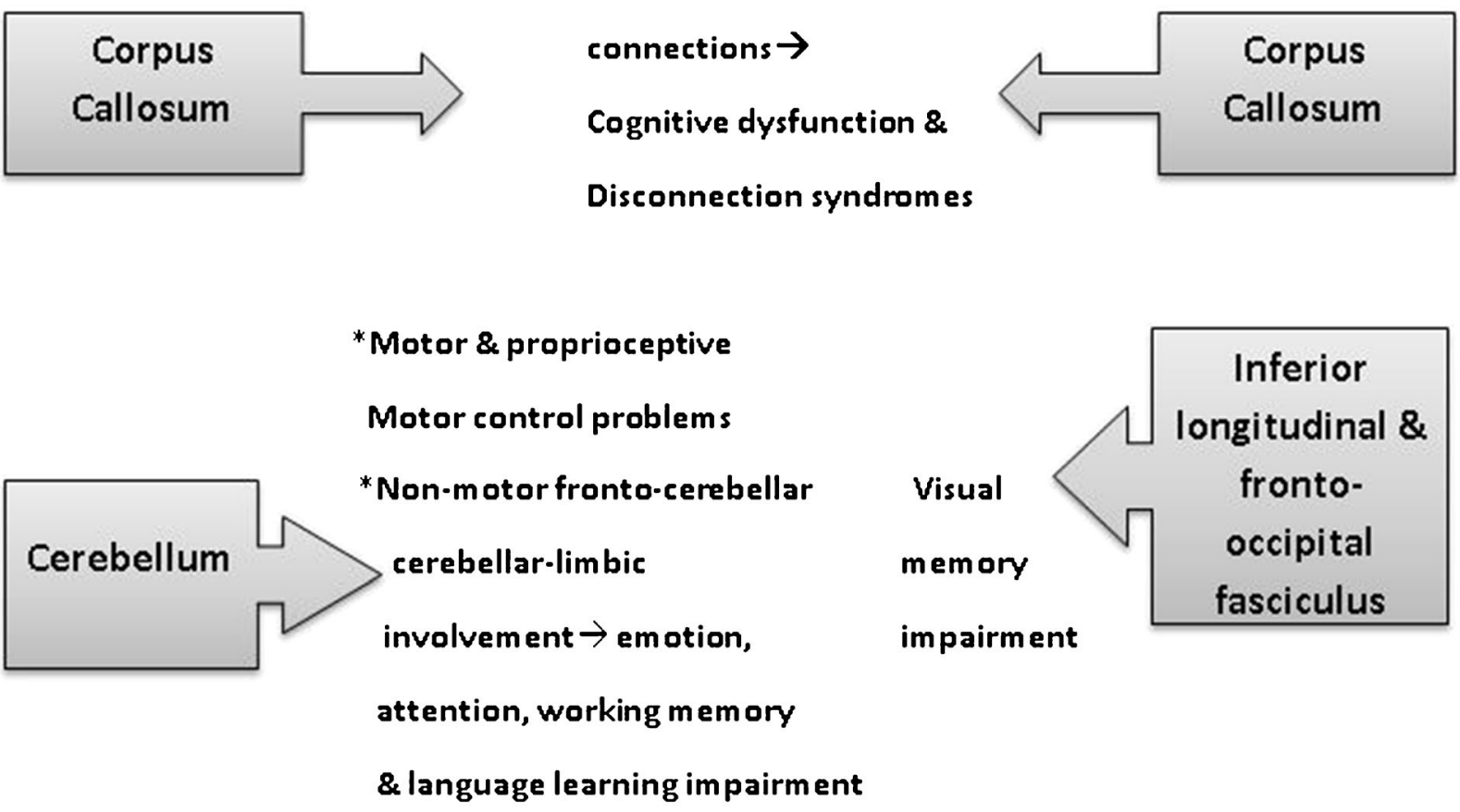

impairment

\section{fasciculus}

Fig. 4 Summary of the most affected regions through volumetric and diffusion tensor imaging (DTI) analyses in the present study and the possible outcomes of these results 
diseases such as schizophrenia, autism and the evidence of neuropsychological abnormalities including memory impairment due to damage in genu and ALIC have been reported before [34-36]. Therefore, our findings on WM degradation in the ALIC may indicate the reduced function of the prefrontal cortex for executive function and spatial working memory in patients with DS. The impairment of thalamocortical fibres in the ALIC may affect the crucial connectivity of visual, attentional and somatosensory systems in patients with DS [37].

Another important aspect of frontal circuits is involvement of the UF which is one of the major long-range WM tracts that connects the orbitofrontal cortex to the anterior temporal lobes. It is assumed that the functions should align associative and episodic memory, linguistic and social-emotional functions due to typically relating with the limbic system. Deficits of short-term and episodic memory tasks and reductions in fMRI signals on the medial temporal lobe may be related to damage to the UF [38-40]. The FA decrease in UF may represent an earlier stage of hippocampal damage and reflect the affected cellular integrity with functional deficits.

Many individuals with DS face the daily challenge of negatively affected visual object memory [41]. The ILF is a ventral associative bundle with considerable functions in visual perception and object recognition. It connects the occipital lobe to the anterior part of the temporal lobe that runs above the optic pathways and overlaps with the IFOF, which is another direct pathway that connects the occipital, orbito-frontal and posterior-temporal areas. On the other hand, the ILF is linked to the UF so that it can transfer information to the orbitofrontal brain. In our study, degradation of integrity of the ILF and IFOF with decreased FA values may indicate visual memory impairment consistent with Ortibus et al. [42], who have reported a relationship between ILF and IFOF integrity in children with visual- perceptual impairment [42].

Finally, decreased FA in the CC, the largest WM structure that facilitates interhemispheric communication, provides significant evidence of WM involvement in DS. Lower FA values are consistent with the recent DTI study in adults by Powell et al. [25] and showed a positive correlation with decreased volumetry in our study. Cognitive dysfunction and several disconnection syndromes may result from the impaired integrity of the CC. The positive relationship between the UF and CCminF may indeed reflect functional similarity as well as spatially sharing overlapping paths.

$\mathrm{MD}$ and FA are complementary measurements of tissue damage. In our study, the UF, ILF, IFO, right-CP and ALIC showed decrements in FA accompanied by increments in MD, presenting the major affected fibres of WM in DS. However, independent MD increments in the clusters of the external capsule, anterior corona radiata and thalamic radiatio may demonstrate that diffusion is increased subjectively but the cellular integrity of these tissue is not significantly affected.
The FA increment is an uncommon finding during neurodegenerative processes. However, increased FA values have been reported in autism and attention-deficit/hyperactivity disorder [43, 44] before. Thus, we can infer that the initially defective myelinated nerve sheaths of children with DS can show excessive hyperplasia during the overgrowth phase of brain development and lead to a high FA value of WM, whereas this extreme myelination may not completely compensate the defective cognitive function of DS. As previously reported, this condition may also be related to brain compensatory mechanisms of synaptic plasticity [45-47], even if the function is not well preserved.

The most affected regions through volumetric and DTI analyses in the present study and the possible outcomes of these results are summarized in Fig. 4.

\section{Limitations}

The present study has some limitations in that the cognitive/ motor functions of patients were not evaluated and the crosssectional study method involved a small patient group using 1.5-T MRI.

\section{Conclusion}

The neurological deficits of DS appearing from childhood should be investigated for potential treatment strategies. To our knowledge, the preliminary results of WM alterations in children with DS in the present study may define impaired integrity of several fibres and may be related to executive function, visual and spatial memory, and disconnection syndromes. Additionally, as the first neuroimaging volumetric data in such a young patient group with DS, the results may suggest that cerebellar, CC, cortical, subcortical and deep GM volume reductions begin as early as 2 years of age. The lack of brain segmental volume reduction with more extensive WM impairment in this age group may suggest that WM degeneration may be an early step in neurodevelopmental delay. In future studies, combining DTI and functional MRI in larger patient groups will contribute to providing further details about tissue microstructure and function of DS in neurodevelopmental and neurodegenerative progress.

Acknowledgements This paper was presented as an oral presentation at ECR 2016. The scientific guarantor of this publication is Hediye Pinar Gunbey. The authors of this manuscript declare no relationships with any companies whose products or services may be related to the subject matter of the article. The authors state that this work has not received any funding. Aslihan Alhan kindly provided statistical advice for this manuscript. Institutional Review Board approval was obtained. Written informed consent was obtained from all subjects (patients) in this study. Methodology: prospective, cross-sectional study, performed at one institution. 


\section{References}

1. Parker SE et al (2010) Updated national birth prevalence estimates for selected birth defects in the United States, 2004-2006. Birth Defects Res Part A: Clin Mol Teratol 88:1008-1016

2. Martin GE et al (2009) Language characteristics of individuals with Down syndrome. Top Lang Disord 29:112

3. Silverman W (2007) Down syndrome: cognitive phenotype. Ment Retard Dev Disabil Res Rev 13:228-236

4. Jarrold C, Baddeley AD, Hewes AK (2000) Verbal short-term memory deficits in Down syndrome: a consequence of problems in rehearsal? J Child Psychol Psychiatry 41:233-244

5. Dodd B (1975) Recognition and reproduction of words by down's syndrome and non-down's syndrome retarded children. Am J Ment Defic 80:306-311

6. Fowler A, Gelman R, Gleitman LR (1994) The course of language learning in children with Down syndrome. In: Tager-Flusberg $\mathrm{H}$ (ed) Constraints on language acquisition studies of atypical children. NJ Lawrence Erlbaum Associates, Hillsdale

7. Baddeley A, Jarrold C (2007) Working memory and Down syndrome. J Intellect Disabil Res 51:925-931

8. Carr J (1970) Mental and motor development in young mongol children*. J Intellect Disabil Res 14:205-220

9. Engidawork E, Lubec G (2003) Molecular changes in fetal Down syndrome brain. J Neurochem 84:895-904

10. Guihard-Costa A-M et al (2006) Biometry of face and brain in fetuses with trisomy 21. Pediatr Res 59:33-38

11. Jernigan TL, Bellugi U (1990) Anomalous brain morphology on magnetic resonance images in Williams syndrome and Down syndrome. Arch Neurol 47:529-533

12. Pinter $J$ et al (2001) Amygdala and hippocampal volumes in children with Down syndrome: a high-resolution MRI study. Neurology 56:972-974

13. Kates WR et al (2002) Cerebral growth in fragile $X$ syndrome: review and comparison with Down syndrome. Microsc Res Tech 57:159-167

14. Śmigielska-Kuzia J et al (2011) A volumetric magnetic resonance imaging study of brain structures in children with down syndrome. Neurol Neurochir Pol 45:363-369

15. Onorati P et al (2013) Whole-brain voxel-based morphometry study of children and adolescents with down syndrome. Funct Neurol 28:19

16. Condoluci C, Onorati P, Albertini G (2009) Gait analysis and cerebral volumes in down's syndrome. Funct Neurol 24:147

17. Menghini D, Costanzo F, Vicari S (2011) Relationship between brain and cognitive processes in down syndrome. Behav Genet 41:381-393

18. Lee NR, Adeyemi EI, Lin A et al (2015) Dissociations in cortical morphometry in youth with down syndrome: evidence for reduced surface area but increased thickness. Cereb Cortex 26:2982-2990

19. Adeyemi EI, Giedd JN, Lee NR (2015) A case study of brain morphometry in triplets discordant for down syndrome. Am J Med Genet A 167:1107-1110

20. Fischl B (2012) FreeSurfer. Neuroimage 62:774-781

21. Nelson L et al (2005) Learning and memory as a function of age in down syndrome: a study using animal-based tasks. Prog NeuroPsychopharmacol Biol Psychiatry 29:443-453

22. Lott IT, Dierssen M (2010) Cognitive deficits and associated neurological complications in individuals with down's syndrome. Lancet Neurol 9:623-633

23. Basser PJ, Pierpaoli C (2011) Microstructural and physiological features of tissues elucidated by quantitative-diffusion-tensor MRI. J Magn Reson 213:560-570

24. Alexander AL et al (2007) Diffusion tensor imaging of the brain. Neurotherapeutics 4:316-329

25. Powell D et al (2014) Frontal white matter integrity in adults with down syndrome with and without dementia. Neurobiol Aging 35: 1562-1569
26. Fischl B et al (2004) Sequence-independent segmentation of magnetic resonance images. Neuroimage 23:S69-S84

27. Romano A et al (2015) Age effects on cortical thickness in young down's syndrome subjects: a cross-sectional gender study. Neuroradiology 57:401-411

28. Esposito $\mathrm{G}$ et al (2008) Genomic and functional profiling of human down syndrome neural progenitors implicates S100B and aquaporin 4 in cell injury. Hum Mol Genet 17:440-457

29. Karlsen AS, Pakkenberg B (2011) Total numbers of neurons and glial cells in cortex and basal ganglia of aged brains with down syndrome - a stereological study. Cereb Cortex 21:2519-2524

30. Anderson JS et al (2013) Abnormal brain synchrony in down syndrome. NeuroImage: Clin 2:703-715

31. Teipel SJ et al (2003) Relation of corpus callosum and hippocampal size to age in nondemented adults with down's syndrome. Am J Psychiatr 160:1870-1878

32. Jeringan T, Bellugi U, Sowell E (1993) Cerebral morphological distinctions between William's and down's syndromes. Arch Neurol 50:186-191

33. Strick PL, Dum RP, Fiez JA (2009) Cerebellum and nonmotor function. Annu Rev Neurosci 32:413-434

34. Chukwudelunzu F et al (2001) Extensive metabolic and neuropsychological abnormalities associated with discrete infarction of the genu of the internal capsule. J Neurol Neurosurg Psychiatry 71:658-662

35. Rosenberger $\mathrm{G}$ et al (2012) Anterior limb of the internal capsule in schizophrenia: a diffusion tensor tractography study. Brain Imaging Behav 6:417-425

36. Shukla DK et al (2010) White matter compromise of callosal and subcortical fiber tracts in children with autism spectrum disorder: a diffusion tensor imaging study. Journal of the American Academy of Child \& Adolescent Psychiatry 49:1269-1278, e2

37. Guillery R, Sherman SM (2002) Thalamic relay functions and their role in corticocortical communication: generalizations from the visual system. Neuron 33:163-175

38. Anderson JS et al (2015) Violence: heightened brain attentional network response is selectively muted in down syndrome. $\mathrm{J}$ Neurodev Disord 7:1

39. Carlesimo GA, Marotta L, Vicari S (1997) Long-term memory in mental retardation: evidence for a specific impairment in subjects with down's syndrome. Neuropsychologia 35:71-79

40. Frenkel S, Bourdin B (2009) Verbal, visual, and spatio-sequential short-term memory: assessment of the storage capacities of children and teenagers with down's syndrome. J Intellect Disabil Res 53: $152-160$

41. Vicari S, Bellucci S, Carlesimo GA (2006) Evidence from two genetic syndromes for the independence of spatial and visual working memory. Dev Med Child Neurol 48:126-131

42. Ortibus $\mathrm{E}$ et al (2012) Integrity of the inferior longitudinal fasciculus and impaired object recognition in children: a diffusion tensor imaging study. Dev Med Child Neurol 54:38-43

43. Bashat DB et al (2007) Accelerated maturation of white matter in young children with autism: a high b value DWI study. Neuroimage 37:40-47

44. Li Q et al (2010) Increased fractional anisotropy in white matter of the right frontal region in children with attention-deficit/hyperactivity disorder: a diffusion tensor imaging study. Neuroendocrinol Lett $31: 747$

45. Engvig A et al (2012) Memory training impacts short-term changes in aging white matter: a longitudinal diffusion tensor imaging study. Hum Brain Mapp 33:2390-2406

46. Tavor I, Hofstetter S, Assaf Y (2013) Micro-structural assessment of short term plasticity dynamics. Neuroimage 81:1-7

47. Chan $\mathrm{KC}$ et al (2011) In vivo manganese-enhanced MRI and diffusion tensor imaging of developing and impaired visual brains. In: 2011 Annual International Conference of the IEEE Engineering in Medicine and Biology Society. IEEE. p. 7005-7008 